\section{Premorbid, psychosocial and clinical predictors of the costs of schizophrenia and other psychoses}

\author{
VAUGHAN J. CARR, TERRY J. LEWIN, AMANDA L. NEIL, SEAN A. HALPIN \\ and SCOTT HOLMES
}

The cost of mental health care for schizophrenia is high, being $2-3 \%$ of the total health and social services expenditure (Knapp, 1997). In Australia, treatment costs for the major psychotic disorders have been estimated at $2.8 \%$ of the health and community services expenditure, with schizophrenia and schizoaffective disorder together accounting for 2.0\% (Carr et al, 2002, 2003a). Although the main component of mental health care costs for schizophrenia is hospitalisation (Lang et al, 1997), there are also substantial indirect or time-loss costs. If factors that are predictive of higher treatment and/or indirect costs can be identified, they may be useful as foci for interventions aimed at reducing costs. For example, lower levels of functioning have been associated repeatedly with higher treatment costs (e.g. Rund \& Ruud, 1999; Knapp et al, 2002). Other premorbid, psychosocial and clinical factors have been associated less consistently with treatment costs, and indirect costs rarely have been examined in this context. The purpose of this paper is to identify predictors of treatment costs and indirect costs in psychotic disorders and to discuss their potential for guiding cost-saving interventions.

\section{METHOD}

\section{Overview}

Conclusions Several concurrent strategies were recommended, including early intervention programmes and assertive evidence-based rehabilitation and supported employment programmes aimed at reducing disability. The costeffectiveness of these approaches needs to be evaluated from the perspectives of both government and society.

Declaration of interest None.

\section{Sample}

The LPDS sample was obtained from a two-phase, census-based study conducted in four metropolitan areas in 1997-1998. The inclusion criteria were: age 18-64 years; and an ICD-10 diagnosis (World Health Organization, 1992) of any non-organic or non-substance-induced psychotic disorder. Phase 1 comprised a 1month census of all individuals in contact with 'mainstream' mental health services in the four participating areas. This sample was supplemented by patients drawn from the case-loads of general practitioners or private psychiatrists in the participating areas, persons of no fixed abode or living in marginal accommodation and persons with previous service contacts but not in contact with the services in the census month. All eligible consenting individuals were screened for psychosis using a set of six questions targeting psychotic symptoms (Jablensky et al, 2000). Phase 2 comprised standardised interviews with a stratified random sample $(n=980)$ of the screenpositive individuals $(n=3797)$. Exclusion criteria for the study were: temporary visitor status in Australia; significant cognitive deficit; residence in a nursing home or prison; and inability to communicate adequately in English. Approval for the study was obtained from the relevant institutional ethics committees.

\section{Measures}

The post-screening assessment instrument was the Diagnostic Interview for Psychosis (DIP), a semi-structured diagnostic interview comprising three modules: demographic and social functioning, including selected items from the World Health Organization (WHO) Disability Assessment Schedule (DAS; World Health Organization, 1988); diagnosis using the Operational Criteria for Psychosis (OPCRIT; McGuffin et al, 1991) and elements of the WHO Schedules for Clinical Assessment in Neuropsychiatry (SCAN; Wing et al, 1990); and reported usage of a range of hospital- and community-based services in the past year. Interviews were conducted by trained clinical interviewers, for whom there was a satisfactory level of interrater diagnostic agreement (generalised $\kappa=0.73$ for ICD-10 diagnoses).

Among the interview items was a global rating of the course of illness, which required the interviewer to use all available information to assign the participant to 
one of five categories: 'single episode, with good or unknown recovery'; 'multiple episodes, with good recovery between episodes'; 'multiple episodes, with partial recovery between episodes'; 'continuous chronic illness, with little or no deterioration'; or 'continuous chronic illness, with clear deterioration'. Participants also reported their extent of service use during the previous 12 months in the following categories: in-patient hospitalisation (psychiatric and non-psychiatric); out-patient services (psychiatric and non-psychiatric), which included attendances at community mental health clinics or receiving home visits; and emergency service contacts (psychiatric and non-psychiatric), which included the use of community-based mental health crisis teams. Use of psychiatric rehabilitation services and consultations with psychiatrists and psychologists in private practice and with general practitioners were also recorded, together with the medications used (Carr et al, 2003b). Participants also were asked to specify if they had had any need over the previous 12 months for a particular kind of service that they were unable to access (i.e. 'unmet need').

Three measures of disability were derived from the interview data. First, two disability scales were constructed based on item loadings from a principal components analysis of the DAS: a personal disability score (range 0-10) that covered five DAS items (participation in household activities, interests, self-care, occupational performance and overall socialising); and a social disability score (range 0-6) that included three DAS items (intimate relationships, deterioration in relationships and social withdrawal). Second, we regrouped global ratings (range 0-10) from the Social and Occupational Functioning Assessment Scale (SOFAS; American Psychiatric Association, 1994), with higher scores indicating better functioning. The DIP items covering current symptoms and mental state, and symptoms during the previous year, were also subjected to a principal components analysis to confirm their patterns of association. Based on the item loadings, scores on four symptom factors were derived: depression (range 0-20); mania (range 0-9); reality distortion (range 0-16); and disorganisation (range $0-8$ ). To facilitate comparisons with other studies, a negative-symptom score (range $0-3)$ was also derived by grouping three of the items (restrictive affect, blunted affect, negative formal thought disorder) that otherwise would have been included in the disorganisation factor.

\section{Cost estimation}

The current analysis was undertaken from the perspectives of government and society, the former referring to the financial costs of psychosis incurred by governments, both state and national, and the latter providing an overall estimate of the opportunity costs associated with psychotic disorders. Within both perspectives the costs were considered to fall into three broad categories: direct mental health care costs (e.g. associated with health professionals, in-patient and community treatment, medication and rehabilitation programmes); indirect or time-loss costs, which included transfer payments (e.g. pensions and other income support) and tax foregone (government perspective) and patient and carer earnings foregone (societal perspective); and other sector costs (e.g. accommodation support, legal and other administrative costs and voluntary sector costs). The general costs of providing health care that could not be regarded as psychosis-related were estimated separately but excluded from the current analyses.

Individual costs were estimated by multiplying the measured quantities of services and other resources utilised by their unit price. However, not all services and agencies were recorded in detail. For example, utilisation of services provided by nongovernment or voluntary organisations (e.g. support groups, charities) as well as government social and welfare agencies were noted, but the number and types of services utilised were not recorded. However, conservative estimates of costs incurred within this sector have been made. In other instances, such as medication use, where only the identity of resources used was captured, conservative assumptions have been made to obtain an estimate of resource use.

A set of standard (and conservative) unit prices in Australian dollars (AUS\$) was employed to value resource consumption. Costs were estimated for the year 2000 (average exchange rate: AUS $\$ 1.00=\mathrm{UK} £ 0.3836$ ). Indirect costs were calculated on the basis of the traditional human capital approach, which estimates 'potential' production losses, as opposed to the 'friction cost' approach, which uses 'actual' production losses (Koopmanschap et al, 1995). We have adopted the position advocated by Weinstein et al (1997) that the friction cost approach does not take into account the full costs of lost productivity, only the social cost of employment transition. The specific methodologies and assumptions employed in the calculation of costs are detailed elsewhere (Carr et al, 2002) and further information is available from the authors on request. From the individual costs, the average cost per person with treated psychosis was calculated.

\section{Statistical analysis}

In the data analyses three cost estimates were considered, each calculated from government and society perspectives: direct mental health care costs; indirect or time-loss costs; and total costs (which also included 'other sector' costs). Analyses of differences between groups were based on analysis of variance (continuous variables) or overall $\chi^{2}$ tests (categorical variables). The major analyses comprised a series of five-step hierarchical regressions in which the outcome variables were the aggregate cost estimates. Bonferroni-adjusted $(\alpha / k)$ family-wise error rates were used to control for the number of statistical tests within families.

In view of recent concerns about the appropriateness of particular regression models for examining mental health care costs (Dunn et al, 2003), we offer the following rationale for our approach. Hierarchical regression procedures have a well-established role in psychosocial research in that they facilitate an ordering of predictors according to a presumed causal priority, they allow for the inclusion of potential confounding factors and they permit the testing of key researcher-determined hypotheses (Cohen \& Cohen, 1983). Importantly, the predictor variables in the current hierarchical regression analyses were chosen carefully to avoid factors that had contributed directly to the calculation of costs (e.g. hospitalisation, medication, service utilisation, employment and welfare status variables) and comprised most of the non-redundant premorbid, psychosocial and clinical variables available in the study. The primary focus of these regressions was the identification of individual predictor variables making an independent contribution to prediction, with partial correlations ( $p r)$ being the preferred choice for reporting the magnitude of effects. For descriptive purposes, incremental variance estimates are also reported for each step in the hierarchy. Costs 
data are often highly skewed but this was less so in the current data-set, probably owing to the sampling procedures (e.g. selection of patients with similar diagnoses in recent contact with health services) and the nature of the aggregate cost indices. In any event, we prefer raw (i.e. untransformed) costs data, as do others (e.g. Lumley et al, 2002; Dunn et al, 2003), and believe that conventional linear regressions are sufficiently robust to violation of their assumptions that they should be regarded as the default approach, particularly when sample sizes are large (e.g. Lumley et al, 2002).

\section{RESULTS}

\section{Sample characteristics}

The distribution of ICD-10 diagnoses was: schizophrenia, $510(52.0 \%)$; schizoaffective disorder, $102(10.4 \%)$; bipolar disorder, mania, 112 (11.4\%); depressive psychosis, 67 (6.8\%); other ICD-10 psychotic disorders, 145 (14.8\%); and subthreshold disorders for ICD-10 that met the psychosis criteria in other diagnostic systems, 44 (4.5\%). For convenience, the findings are reported in terms of two diagnostic groups: schizophrenia $(n=510)$ and 'other psychoses' $(n=470)$. Table 1 reports the overall characteristics of the sample, together with a breakdown by diagnosis and associated statistical comparisons.

As shown in Table 1, the sample was approximately $60 \%$ male with a mean age of 39 years. There was a high rate of failure to complete high school, most participants had never been married, most were currently unemployed and almost one-fifth reported a family history of schizophrenia. Illness onset tended to be in the low- to mid-20s and duration of illness was around 15 years, with approximately $70 \%$ experiencing either a chronic course of illness or multiple episodes with only partial recovery between episodes. Almost half of the sample had had at least one psychiatric hospitalisation in the past year, among whom the mean aggregate length of stay was approximately 2 weeks. There were relatively high lifetime rates of substance misuse/dependence and typically high rates of current tobacco smoking. Overall, the participants were moderately to severely disabled, as reflected in their mean SOFAS scores.

Participants with schizophrenia differed significantly from those with other psychoses on a range of demographic and illness-related variables (see Table 1). For example, they were more likely to be male, less likely to have completed high-school education or to have been married, more likely to be unemployed and tended to have a more chronic illness course with greater disability. Although the two groups had similar lifetime histories of substance misuse/ dependence, patients with schizophrenia were more likely to be current smokers and, if hospitalised, tended to have spent more days in hospital during the previous year.

\section{Aggregate cost estimates}

Estimated annual costs of psychosis per patient are summarised in Table 2. On average, each treated patient with psychosis cost the Australian government AUS\$29600 (UK£11 355) per annum, whereas the corresponding societal costs were estimated to be AU\$46200 (UK£17 722) per annum. Carr et al $(2002,2003 a)$ present detailed breakdowns of these costs, together with weighted prevalence-based estimates of the total annual population costs for urban Australia. Annual aggregate cost estimates per patient were higher among the schizophrenia group than among those with other psychoses (see Table 2).

\section{Predictors of the costs of psychosis}

Prior to undertaking the major analyses, we examined simple correlations among the six cost indices in Table 2. Mental health care cost estimates from the government and society perspectives and the total cost estimates from the government perspective were all highly intercorrelated $(r \geqslant 0.98)$. Consequently, only four cost indices were retained in the prediction analyses: mental health care costs (from either perspective), indirect costs from the government and society perspectives $(r=0.60)$, and total costs from a society perspective. These four outcome variables were regressed onto the 43 continuous and contrast-coded (categorical) predictor variables shown in Table 3, which were grouped according to a predetermined (pseudo-chronological) five-step hierarchy. It should be noted that psychosis diagnosis, expressed as the contrast between schizophrenia (1) and other psychoses $(-1)$, was included in step 4 of the hierarchy. Although this contrast was not significant in any of the regression analyses, there were univariate associations with each of the outcomes; the simple associations $(r)$ between the psychosis diagnosis contrast and the four outcome variables in Table 4 were $0.15,0.20,0.13$ and 0.19 , respectively.

The statistically significant predictors from the five-step hierarchical regression analyses are shown in Table 4, together with the increments in explained variance associated with each step. The set of 43 predictor variables accounted for $30.2 \%$ of the variance in total annual societal costs per patient. The explained variance was lower for mental health care costs $(23.9 \%)$ and higher for government indirect costs $(38.6 \%)$, owing mainly to the relative contributions of predisposing factors (step 1: $3.1 \% v .20 .5 \%$ ). By comparison, the lower overall explained variance in societal indirect costs $(21.6 \%)$ was due mainly to the reduced contributions of illness onset and course-related factors (step 3 ) and current symptoms and disability factors (step 5). Because most of the significant predictors were associated with multiple outcome variables, with similar patterns of association $(p r)$, the findings are described below on a step-by-step basis rather than separately for each cost estimate.

Among the predisposing factors (step 1), failure to complete high school was the most robust predictor, being significantly associated with higher costs on all four indices. Male gender was associated most strongly with indirect costs from the government perspective, but also with societal indirect and total costs. Age was associated positively with indirect costs from both perspectives, and participants from nonEnglish speaking backgrounds also tended to have higher societal indirect costs. With the predisposing variables controlled, of the family and support factors (step 2), being previously (but not currently) married was associated with higher indirect costs from the government perspective but a higher availability of friends was associated with lower societal indirect and total costs. Illness onset and course-related factors (step 3) contributed an additional $4.6-11.9 \%$ of the explained variance, after controlling for the foregoing sets of predictors. Chronicity of illness course was a significant predictor in all categories of costs. Earlier age at onset made a small but significant contribution to mental health care costs and indirect costs from a government perspective, whereas dissatisfaction with one's own independence was associated with mental health care and total costs. 


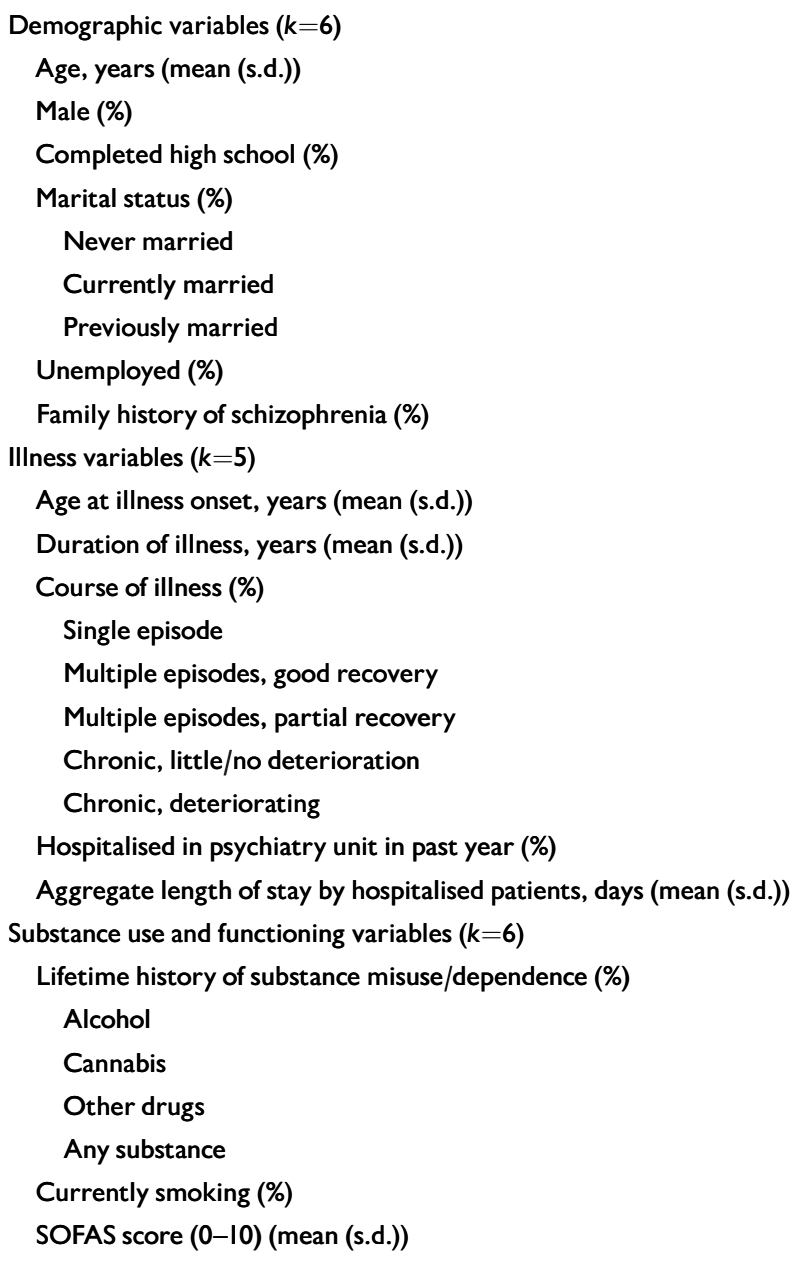

Unemployed (\%)

Family history of schizophrenia (\%)

Illness variables $(k=5)$

Age at illness onset, years (mean (s.d.))

Duration of illness, years (mean (s.d.))

Course of illness (\%)

Single episode

Multiple episodes, good recovery

Multiple episodes, partial recovery

Chronic, little/no deterioration

Chronic, deteriorating

Aggregate length of stay by hospitalised patients, days (mean (s.d.))

Substance use and functioning variables $(k=6)$

Lifetime history of substance misuse/dependence (\%)

Alcohol
Cannabis
Other drugs
Any substance
Currently smoking (\%)
SOFAS score $(0-10)($ mean (s.d.))

40.12 (11.85)
$51.9 * * *$

57.0***

39.25 (11.6I)

$\begin{array}{ll}53.4^{* * *} & 63.5 \\ 21.9 & 15.9 \\ 24.7 & 20.6 \\ 66.2^{* * *} & 72.0 \\ 14.9 & 18.0\end{array}$

$\begin{array}{ccc}28.4 & 31.7 & 30.0 \\ 27.8 & 22.1 & 25.1 \\ 13.1 & 13.2 & 13.2 \\ 42.9 & 40.4 & 41.7 \\ 72.8 & 64.0^{*} & 68.6 \\ 5.33(1.68) & 5.83(1.54)^{* * *} & 5.57(1.63)\end{array}$

24.17 (8.84) $15.07(10.90)$

SOFAS, Social and Occupational Functioning Assessment Scale.

I. Comparisons between groups were based on analysis of variance (continuous variables) or overall $\chi^{2}$ tests (categorical variables). Bonferroni-adjusted ( $\left.\alpha / k\right)$ family-wise probabilities were used, controlling for the $k$ variables per family: $* P<0.05 ; * * * P<0.001$.

Table 2 Estimated annual costs of psychosis per patient

\begin{tabular}{|c|c|c|c|}
\hline \multirow[t]{2}{*}{ Perspective and cost category } & \multicolumn{3}{|c|}{ Average annual costs per patient (AUS\$) by diagnostic group' } \\
\hline & Schizophrenia $(n=510)$ & Other psychoses ${ }^{2}(n=470)$ & Overall $(n=980)$ \\
\hline \multicolumn{4}{|l|}{ Government perspective } \\
\hline Mental health care costs & 21300 & $12200 * * *$ & 17200 \\
\hline Indirect or time-loss costs & 13000 & $10300 * * *$ & 11700 \\
\hline Total costs ${ }^{3}$ & 35000 & $23000 * * *$ & 29600 \\
\hline \multicolumn{4}{|l|}{ Society perspective } \\
\hline Mental health care costs & 21600 & $13000 * * *$ & 17800 \\
\hline Indirect or time-loss costs & 29000 & $25800 *$ & 27500 \\
\hline Total costs ${ }^{3}$ & 51600 & $39500 * * *$ & 46200 \\
\hline
\end{tabular}

I. Weighted cost estimates in year 2000 Australian dollars (AUS\$), adjusted to compensate for recruitment biases; figures have been rounded to the nearest $\$ 100$; average exchange rate: AUS $\$ 1.00=U K £ 0.3836$.

2. Comparisons between groups were based on weighted analyses of covariance (with gender as the covariate). Bonferroni-adjusted $(\alpha / k)$ probabilities were used, controlling for the $k=6$ cost variables: $* P<0.05 ; * * * P<0.001$. 3. Total costs include mental health care costs, indirect or time-loss costs (e.g. pension payments and tax foregone from a government perspective and earnings foregone from a society perspective) and other sector costs (e.g. accommodation support, legal and other administrative costs).
Diagnosis and lifetime substance misuse variables (step 4) were not significant predictors of any costs, after controlling for the preceding predictors in the hierarchy. Finally, current symptoms and disablement factors (step 5) accounted for an additional $4.9-9.6 \%$ of the explained variance. In particular, reality distortion and disorganisation symptoms, personal disability and recent suicide or self-harm attempts each contributed significantly to higher mental health care and total costs, whereas frequency of current alcohol consumption contributed to reduced levels of these costs. Lower levels of depression, greater impairment due to medication side-effects and higher cigarette consumption were associated with indirect costs from the government perspective but not with total costs or any of the other cost indices. Overall, 
Table 3 The 43 predictor variables used in the five-step hierarchical regression analyses

\section{Step $(m=$ number of predictors) Predictor variables}

I. Predisposing factors $(m=9)$

Age; gender; premorbid work adjustment; premorbid social adjustment; premorbid personality disorder; highschool education completion; family history of schizophrenia; family history of other psychiatric disorders; main language spoken at home

2. Family and support factors $(m=5) \quad$ Marital status, never v. ever married; marital status, previously v. currently married; face-to-face family support; availability of friends; number of children

3. Illness onset and course-related factors Age at onset; mode of onset; psychosocial stressor prior to onset; coarse brain disease prior to onset; course of $(m=7)$ illness (single episode and good or unknown recovery, multiple episodes and good recovery, multiple episodes and partial recovery, chronic illness and little or no deterioration, chronic illness and clear deterioration); dissatisfaction with own independence; dissatisfaction with life as a whole

4. Diagnosis and lifetime substance use factors $(m=7)$

Diagnosis, schizophrenia v. other psychoses; lifetime alcohol misuse/dependence; lifetime cannabis misuse/ dependence; lifetime other substance misuse/dependence; frequency of cannabis use at worst period ever; frequency of amphetamine use at worst period ever; frequency of other substance use at worse period ever

5. Current factors: symptoms (current or Depression; mania; reality distortion; disorganisation; negative symptoms; personal disability; social disability; present in the past year), disability, SOFAS score; any criminal charges during past year; victim of violence or felt unsafe during past year; recent functioning, current substance use and suicide or self-harm attempts; impairment due to medication side-effects; unmet need; current cigarette unmet need $(m=15)$ consumption per day; frequency of current alcohol consumption

SOFAS, Social and Occupational Functioning Assessment Scale.

higher current functioning (i.e. SOFAS score) was associated with lower indirect and total costs but not with direct mental health care costs.

\section{Predictors of the costs of schizophrenia}

These regression analyses were repeated for the subsample of participants with an ICD10 diagnosis of schizophrenia $(n=510)$. In broad terms, the pattern of results was consistent with that reported in Table 4, with corresponding explained variance estimates of $29.3 \%, 37.9 \%, 25.1 \%$ and $35.4 \%$, respectively. However, within this subsample, approximately one-third of the predictors were no longer statistically significant. Specifically, first language (step 1), marital status, availability of friends (step 2), reality distortion and disorganisation symptoms, personal disability, medication side-effects and recent suicide or self-harm attempts (step 5) were not associated significantly with any of the cost indices. In addition, gender was no longer associated with total societal costs, and high-school education status was not associated with indirect societal costs. Greater homogeneity within the schizophrenia subsample may have contributed to these effects (e.g. they tended to be unemployed males, who had never married, with a more chronic illness course, see Table 1). On the other hand, there were three significant associations that were not found in the previous analyses (Table 4): earlier age at illness onset (step 3) was associated significantly with total societal costs $(p r=-0.11$, $P<0.05$ ), whereas an unmet need for services (step 5) was associated significantly with mental health care costs $(p r=0.13$, $P<0.05)$ and total societal costs ( $p r=0.12, P<0.05$ ).

\section{DISCUSSION}

\section{Findings}

Overall, the costs of schizophrenia were greater in all cost categories than the costs of the 'other' psychotic disorders (see Table 2), reflecting the higher levels of disadvantage, chronicity and disablement in schizophrenia (see Table 1). In absolute terms, from the government perspective the largest contributor to the annual costs of psychosis per patient was direct mental health care costs (AUS\$17200 (UK£6598) out of AUS\$29600 (UK£11355), or 58.1\%), whereas from the societal perspective indirect or time-loss costs contributed proportionately more to total costs (AUS $\$ 27500$ (UK£10549) out of AUS\$46200 (UK $£ 17722$ ), or $59.5 \%$ ). The most robust predictors of higher annual psychosis-related costs per patient, across all cost indices, were failure to complete high-school education and chronicity of illness course (see Table 4). Other demographic factors, such as male gender, older age, previously married and lower availability of friends, tended to be associated primarily with higher indirect costs. With the predisposing, support and illness course factors controlled, global ratings of current social and occupational functioning (SOFAS scores) were consistently related to indirect and total costs but not to mental health care costs. On the other hand, mental health care costs during the past year (and associated total costs) were more likely to be linked with illness-related factors (e.g. earlier age of onset, chronicity, reduced independence) and current symptomatology and disability (e.g. reality distortion and disorganisation symptoms, recent suicide or self-harm attempts and reduced ability for personal care).

\section{Consistency with previous research}

The association of lower education levels with higher costs has been noted previously (McCrone et al, 2002) and may reflect several factors (to be discussed subsequently) that contribute to illness characteristics, high service use and unemployment. The robust relationship between course of illness and costs is a unique finding of the present study. Patients with a chronic 
Table 4 Predictors of the annual costs of psychosis per patient $(n=980)$

\begin{tabular}{|c|c|c|c|c|c|c|c|c|c|}
\hline \multicolumn{2}{|c|}{ Step Predictor variables ${ }^{\prime}$} & \multicolumn{8}{|c|}{ Outcome variables ${ }^{2}$} \\
\hline & & \multicolumn{2}{|c|}{ Mental health care costs } & \multicolumn{4}{|c|}{ Indirect costs } & \multirow{2}{*}{\multicolumn{2}{|c|}{$\begin{array}{c}\text { Total costs } \\
\text { Society } \\
\text { perspective }\end{array}$}} \\
\hline & & $(\Delta \operatorname{Var} \%)$ & $\mathrm{pr}$ & \multicolumn{2}{|c|}{$\begin{array}{l}\text { Government } \\
\text { perspective }\end{array}$} & \multicolumn{2}{|c|}{$\begin{array}{c}\text { Society } \\
\text { perspective }\end{array}$} & & \\
\hline & & & & $(\Delta$ Var\%) & pr & $(\Delta$ Var\%) & pr & $(\Delta \operatorname{Var} \%)$ & pr \\
\hline \multirow[t]{5}{*}{$\mathrm{I}$} & Predisposing factors $(m=9)$ & (3.I) & & $(20.5)$ & & (9.3) & & (5.7) & \\
\hline & Age at interview & & & & $0.11 * *$ & & $0.14 * * *$ & & \\
\hline & Gender $($ male $=-I$, female $=I)$ & & & & $-0.35^{* * *}$ & & $-0.18^{* * *}$ & & $-0.10 * *$ \\
\hline & Education (not completed $=-\mathrm{I}$, completed $=\mathrm{I}$ ) & & $-0.13^{* * *}$ & & $-0.21^{* * *}$ & & $-0.13^{* * *}$ & & $-0.17^{* * *}$ \\
\hline & First language $($ English $=-\mathrm{I}$, other $=\mathrm{I})$ & & & & & & $0.08 *$ & & \\
\hline \multirow[t]{3}{*}{2} & Family and support factors $(m=5)$ & $(I . I)$ & & $(2.7)$ & & $(1.8)$ & & (1.9) & \\
\hline & Previously $(-I) v$. currently $(I)$ married & & & & $-0.11 * *$ & & & & \\
\hline & Availability of friends $(0-3)$ & & & & & & $-0.11 * *$ & & $-0.09 *$ \\
\hline \multirow[t]{4}{*}{3} & IIIness onset and course-related factors $(m=7)$ & $(9.7)$ & & (8.3) & & $(4.6)$ & & $(I 1.9)$ & \\
\hline & Age at illness onset & & $-0.09 *$ & & $-0.12^{* * *}$ & & & & \\
\hline & Illness course, chronicity (I-5) & & $0.23 * * *$ & & $0.27 * * *$ & & $0.20 * * *$ & & $0.29 * * *$ \\
\hline & Dissatisfaction with own independence $(0-2)$ & & $0.12 * *$ & & & & & & $0.11 * *$ \\
\hline 4 & Diagnosis and lifetime substance use $(m=7)$ & (I.I) & & $(\mathrm{l} .3)$ & & $(0.9)$ & & $(1.0)$ & \\
\hline \multirow[t]{11}{*}{5} & $\begin{array}{l}\text { Current factors: symptoms, disability, functioning and } \\
\text { unmet need }(m=15)\end{array}$ & $(8.8)$ & & (6.3) & & $(4.9)$ & & $(9.6)$ & \\
\hline & Symptomatology (current or past year) & & & & & & & & \\
\hline & Depression score $(0-20)$ & & & & $-0.14 * * *$ & & & & \\
\hline & Reality distortion (0-16) & & $0.09 *$ & & & & & & $0.09 *$ \\
\hline & Disorganisation score (0-8) & & $0.11 * *$ & & & & & & $0.09 *$ \\
\hline & Disability, personal domain $(0-10)$ & & $0.13^{* * *}$ & & & & & & $0.11 * *$ \\
\hline & Functioning, SOFAS score $(0-10)$ & & & & $-0.19 * * *$ & & $-0.19 * * *$ & & $-0.14 * * *$ \\
\hline & Impairment due to side-effects $(0-3)$ & & & & $0.09 *$ & & & & \\
\hline & Suicide/self-harm attempts (past year) $($ no $=-I$, yes $=I)$ & & $0.10 * *$ & & & & & & $0.09 *$ \\
\hline & Current cigarette consumption per day & & & & $0.13^{* * *}$ & & & & \\
\hline & Frequency of current alcohol consumption & & $-0.09 *$ & & & & & & $-0.09 *$ \\
\hline \multicolumn{2}{|c|}{ Overall variance explained (\%) } & (23.9) & & (38.6) & & $(21.6)$ & & $(30.2)$ & \\
\hline
\end{tabular}

$\Delta$ Var\%, increment in explained variance at each step; pr, partial correlation; SOFAS, Social and Occupational Functioning Assessment Scale.

I. Each five-step hierarchical regression contained 43 predictor variables (see Table 3 ), including continuous measures and contrast-coded variables $(m=$ number of predictors per step); only statistically significant predictors are listed.

2. Only significant partial correlations are shown. Bonferroni-adjusted $(\alpha / k)$ probabilities were used, controlling for the $k=4$ outcome variables: $* P<0.05$; $* * P<0.01$; $* * * P<0.001$.

deteriorating course tend to cost more than those with a chronic non-deteriorating course and those with multiple episodes with variable degrees of recovery, and these in turn cost more than those with a single episode. The relationship of course of illness to indirect costs reflects the impact of disease on employment status, in which time-loss costs due to unemployment make a major contribution to indirect costs.

The association between gender (male) and higher indirect costs, but not direct mental health care costs, contrasts with the findings of others who have reported a relationship between male gender and higher direct costs of schizophrenia (Rund $\&$
Ruud, 1999; Knapp et al, 2002). However, the effect of gender, and to a lesser extent age, on indirect costs is not surprising, given the costing methodology employed (e.g. males tend to earn more and wages generally increase with age). On the whole, the relatively small positive contribution of age to indirect but not direct costs adds little to the current state of contradictory findings regarding the relationship between age, or duration of illness, and costs of schizophrenia (Suleiman et al, 1997; Rund \& Ruud, 1999; Byford et al, 2001; McCrone et al, 2002). The finding of an association between early age of illness onset and costs, while controlling for failure to complete high-school education (and age and gender), indicates the relative independence of these factors in relation to the costs of psychosis. In this context, early age of onset may largely reflect illness duration, but, as noted above, the findings in this area are contradictory. However, there is growing evidence that the severity of the early course of schizophrenia correlates positively with subsequent treatment costs (e.g. Kilian et al, 2003).

The relationship between level of disability (i.e. lower SOFAS score) and costs was expected on the grounds that persons with greater disability are more likely to be high users of a range of health and 
welfare services and to receive income support. This replicates well-established findings in relation to disability in schizophrenia (Rund \& Ruud, 1999; Knapp et al, 2002; McCrone et al, 2002). The fact that overall disability remained a strong predictor of costs, after controlling for predisposing, family and support, onset and course factors, indicates its independent contribution over and above these other factors. In contrast, mental health care costs were related more to symptomatology, recent suicidality or self-harm and associated personal disability (e.g. everyday self-care) than to overall social and occupational functioning. However, based on the findings of Kilian et al (2003), variations in SOFAS scores across occasions may tend to parallel fluctuations in mental health treatment costs.

The association between disorganisation symptoms and direct costs also fails to shed light on the fact that others have variously reported inconsistent findings with respect to general, positive and negative symptoms and the direct costs of schizophrenia (Moscarelli et al, 1991; Lang et al, 1997). However, the association between depression and lower government indirect costs probably reflects the relatively higher levels of functioning among patients who have a psychotic illness with prominent affective symptoms, generally regarded as a good prognostic sign.

The absence of an overall association between costs and lifetime substance use disorders was unexpected. However, the observed association between lower direct and total costs and current alcohol consumption is consistent with that of Laugharne et al (2002). This association may partially reflect accommodation status (e.g. abstinence among those currently hospitalised or living in residential accommodation), poorer engagement with services among current alcohol users or, alternatively, some therapeutic pharmacological benefit from alcohol use in the context of psychosis.

\section{Implications for cost reduction}

Can the results of this study be used to address the issue of reducing the costs of psychosis? The more robust and consistent predictors of costs were failure to complete high-school education, course of illness, overall level of disability, male gender and, especially in relation to schizophrenia, age at onset. If some of these variables have utility in guiding policy or treatment approaches with the potential to reduce costs, it is first necessary to determine what they signify.

Failure to complete school education may indicate onset of the psychosis prodrome, cognitive decline prior to illness onset, declining social adjustment, the effects of substance misuse or other adolescent psychosocial problems (e.g. minor psychiatric morbidity, conduct disturbance, family dysfunction, socio-economic disadvantage). Evidence of underperformance at school beginning at puberty has been reported prior to the onset of schizophrenia some 10 years later (van Oel et al, 2002). However, young people who leave school prematurely are at risk of a number of adverse outcomes in early adulthood, in addition to the psychoses and other mental illnesses (James \& Lawlor, 2001), for example unemployment and poverty (Lamb, 1995), antisocial behaviour and criminal convictions (Fergusson et al, 1997), imprisonment and substance misuse (Mensch \& Kandel, 1988). This is clearly a vulnerable group with high potential to generate downstream costs in a variety of ways. It may be that screening premature school-leavers for risk of psychiatric morbidity, substance misuse, antisocial behaviour and other vulnerability indicators could be built into assessment procedures for employment programmes and the allocation of welfare benefits, and be coupled with early intervention programmes suitable for young people. In addition, early identification of decline in school performance and the emergence of problem behaviours around puberty ought to be taken by teachers as an indication for possible health, psychological and vocational assessments and possible remedial interventions. Appropriate early intervention programmes may be a good investment but they would need to be tested for efficacy and have their cost-effectiveness evaluated, particularly in terms of their potential for long-term cost savings.

The chronicity of the course of psychotic illness presents another challenge for interventions aimed at reducing associated costs. However, the first question to be answered is whether a chronic deteriorating course (constituting almost onequarter of the current sample) can be shifted to one with more of a relapsingremitting pattern or whether the trajectory of psychosis is more or less fixed at the time of onset. Kilian et al's (2003) recent longitudinal analysis of the mid-term costs of schizophrenia found that the strongest predictors were time-invariant characteristics of the patient, with which it would be difficult to intervene. Conversely, interventions to reduce relapse rates and thereby reduce costs have a firm evidence base, but this would not constitute a change in course of illness from one pattern to another as defined in the LPDS. If the course of illness is malleable, to what interventions is it amenable and what is their cost-effectiveness in the short and long term? Again, these are questions for future research.

The issue of disability in social and occupational functioning can be addressed now because there is a range of efficacious psychosocial interventions for improving social functions, as well as vocational rehabilitation programmes with a supported employment focus that have demonstrated efficacy in increasing employment (Twamley et al, 2003). Some of these have proved to be cost-effective but we need more comprehensive evaluations of the long-term cost-effectiveness of the main interventions available in this field.

Early age at onset of psychosis and the relationship between early onset and cost, offers the opportunity for the recent growth in early psychosis detection and intervention programmes (McGorry \& Edwards, 1998) to demonstrate their long-term efficacy and capacity to reduce the downstream costs of psychosis. In addition, can interventions prior to the onset of psychosis prevent or delay onset (e.g. McGorry et al, 2002) and, if so, at what cost and with what savings over subsequent years? How reliably can pre-psychotic individuals be identified, how early would identification need to occur for optimal benefit, what pre-psychotic interventions are appropriate and how cost-effective would they be? These are tantalising questions that need to be tested in the context of the burgeoning early psychosis prevention and intervention movement.

There is a range of methodological and conceptual issues that also need to be addressed. Clearly, the determinants of costs in schizophrenia and other psychoses are complex, as are other assessments of disease burden. Using these determinants as guides for estimating the potential effects of various interventions on these costs is also likely to be complex. For instance, optimal treatments are likely to differ in type and effects, depending on age, gender, level of education, age at onset, illness duration, course, level of disability, social competence, current symptoms and so on. Schizophrenia, let alone psychosis, is not a homogeneous 
entity. Consequently, attempts to model the cost savings or the burden averted by wider implementation of treatments with known efficacy ought to take this heterogeneity into account if errors consequent upon broad approximations and assumed uniformity are to be avoided. For example, Andrews $e t$ al (2003) assumed, based on the Schizophrenia Patient Outcomes Research Team recommendations (Lehman \& Steinwachs, 1998), that optimal antipsychotic drug treatment for schizophrenia entailed universal application of atypical antipsychotics, with clozapine being reserved for the $20 \%$ with treatment resistance. Recent data indicate that atypical antipsychotics are not costeffective for routine use, with the possible exception of risperidone, unless the patient is experiencing moderate to severe sideeffects, and that clozapine is cost-effective for those with a chronic course of illness (43\% in the present sample), especially among those with clear deterioration (A. Magnus, personal communication, 2003). Thus, the Andrews et al's (2003) estimations of burden averted with optimal treatment for schizophrenia ( $22 \%$ overall) may have overestimated the costs of antipsychotic drugs and underestimated the extent of burden avertable by clozapine. Other concerns have been raised about the approach taken by Andrews et al (2003), particularly the nature of their assumptions (Goldberg, 2003) and the need to go beyond short-term symptom change, including consideration of the impacts of assertive community treatment and supported employment (Warner, 2003). It is therefore necessary to be circumspect in interpreting modelling studies such as that of Andrews et al (2003) and, indeed, cross-sectional studies of cost predictors such as the current study. We need to avoid uninformed and incautious policy decisions and become better informed about the broader societal costs, consequences and outcomes of psychosis and its treatment.

\section{Limitations and benefits of the study}

The limitations of this study lie in both the collection of the epidemiological data and the costing process employed. The limitations of the study design have been detailed elsewhere (Jablensky et al, 1999, 2000). However, of particular importance to the current paper, data on service utilisation and treatment were based on the participants' reports at interview and not on actual service records. Although it was considered that variance due to inaccuracies of subjective recall could be expected, there was no reason to suspect major discrepancies between such reports and actual service use (Voruganti et al, 1998), with checks built into the interviews to minimise such distortion (Jablensky et al, 1999). Not all resources were included in the costing process. Further, because fully comprehensive resource utilisation and cost data were not obtained, a number of conservative assumptions had to be made (as detailed in Carr et al, 2002). Briefly, the quantity of resources used had to be estimated in a number of instances, and unit prices were ascribed in all instances. Furthermore, indirect costs were limited primarily to morbidity-related unemployment costs. Consequently, the total costs have been underestimated. Some distortions may also have arisen owing to differing numbers of assumptions required (e.g. only unit costs were assumed in relation to hospitalisation, whereas in terms of medication both quantity and unit costs were assumed).

There are two immediate benefits associated with quantifying service and resource utilisation, and opportunities lost or foregone, in terms of costs. First, this permits the aggregation of a variety of relatively disparate but nevertheless psychosis-related elements and outcomes. Second, the cost metric is readily accepted by health service planners and facilitates comparisons over time (adjusted for inflation) and with varied health systems. However, international comparisons are not necessarily straightforward. Issues to consider include: differences in methodology, in particular differences in the resources costed; differences in relative resource prices between countries and over time; and differences in service availability and accessibility.

Examining the predictors of aggregate cost estimates is clearly more distal than detailed assessments of links between particular psychosocial and clinical factors and specific service profiles (e.g. frequency of service contacts, relapse and readmission rates). Consequently, the partial correlations reported here (see Table 4) may tend to understate the overall predictive value of these factors or, alternatively, highlight the more robust associations. The inclusion of a broader spectrum of disorders (i.e. not just psychoses) could have strengthened the associations between the predictors and health service and indirect costs (e.g. by unmasking effects otherwise hidden by range restriction effects). These limitations notwithstanding, this is the first comprehensive study of predictors of direct, indirect and total costs associated with schizophrenia and other psychoses from the perspectives of government and society. Several robust and consistent predictors of all cost categories have been identified (e.g. failure to complete high-school education, course of illness), as well as specific predictors of mental health care and indirect costs (e.g. age at onset and overall disability, respectively). The potential for cost-reducing interventions that could either be targeted at these predictors or influenced by them has also been discussed.

\section{ACKNOWLEDGEMENTS}

This paper is based primarily on data collected in the framework of the collaborative Low Prevalence Disorders Study (LPDS), an epidemiological and clinical investigation that was part of the National Survey of Mental Health and Wellbeing (NSMHWB), Australia 1997-1998; see Jablensky et al $(1999,2000)$ and Carr et al (2003a) for detailed acknowledgements. We thank the many health professionals who contributed to the LPDS and the NSMHWB and the thousands of Australians who agreed to participate.

\section{REFERENCES}

American Psychiatric Association (1994) Diagnostic and Statistical Manual of Mental Disorders (4th edn) (DSM-IV).Washington, DC: APA

Andrews, G., Sanderson K., Corry, J., et al (2003) Cost-effectiveness of current and optimal treatment for schizophrenia. British Journal of Psychiatry, 183, 427-435.

Byford, S., Barber, J. A., Fiander, M., et al (200I) Factors that influence the cost of caring for patients with severe psychotic illness. Report from the UK 700 trial. British Journal of Psychiatry, 178, 44I-447.

Carr, V., Neil, A., Halpin, S., et al (2002) Costs of Psychosis in Urban Australia. National Survey of Mental Health and Wellbeing: Bulletin 2. Canberra: Publications Production Unit, Australian Commonwealth Department of Health and Ageing. http://www.health.gov.au/ hsdd/mentalhe/resources/reports/costs.pdf

Carr, V. J., Neil, A. L., Halpin, S. A., et al (2003a) Costs of schizophrenia and other psychoses in urban Australia: findings from the Low Prevalence (psychotic) Disorders Study. Australian and New Zealand Journal of Psychiatry, 37, 31-40.

Carr, V. J., Johnston, P. J., Lewin, T. J., et al (2003b)

Patterns of service use among persons with

schizophrenia and other psychotic disorders. Psychiatric Services, 54, 226-235.

Cohen, J. \& Cohen, P. (1983) Applied Multiple Regression/Correlation Analysis for the Behavioral Sciences. Hillsdale, NJ: Lawrence Erlbaum Associates.

Dunn, G., Mirandola, M., Amaddeo, F., et al (2003) Describing, explaining or predicting mental health care costs: a guide to regression models. Methodological review. British Journal of Psychiatry, 183, 398-404.

Fergusson, D. M., Lynskey, M.T. \& Horwood, L. J. (1997) The effects of unemployment on juvenile offending. Criminal Behaviour and Mental Health, 7, 49-68. 
Goldberg, D. (2003) Invited commentary on: Costeffectiveness of current and optimal treatment for schizophrenia. British Journal of Psychiatry, 183, 436.

Jablensky, A., McGrath, J., Herrman, H., et al (1999) People Living with Psychotic Illness: An Australian Study 1997-98. National Survey of Mental Health and Wellbeing: Report 4. National Mental Health Strategy: Canberra: Mental Health Branch, Australian Commonwealth Department of Health and Ageing. http: / wwww. health.gov.au:80/hsdd/mentalhe/ resources/reports/plpi_98.htm

Jablensky, A., McGrath, J., Herrman, H., et al (2000) Psychotic disorders in urban areas: an overview of the methods and findings of the study on low prevalence disorders. Australian and New Zealand Journal of Psychiatry, 34, 221-236.

James, D. \& Lawlor, M. (200I) Psychological problems of early school leavers. Irish Journal of Psychological Medicine, 18, 61-65.

Kilian, R., Matschinger, H., Becker, T., et al (2003) A longitudinal analysis of the impact of social and clinical characteristics on the costs of schizophrenia treatment. Acta Psychiatrica Scandinavica, 107, 351-360.

Knapp, M. (1997) Costs of schizophrenia. British Journal of Psychiatry, I7I, 509-518.

Knapp, M., Chisholm, D., Leese, M., et al (2002) Comparing patterns and costs of schizophrenia care in five European countries: the EPSILON study. Acta Psychiatrica Scandinavica, 105, 42-54.

Koopmanschap, M. A., Rutten, F. F. H., van Ivenveld, B. M., et al (1995) The friction cost method for measuring indirect cost of disease. Journal of Health Economics, 14, 17I-189.

Lamb, S. (1995) Dropping out of school in Australia: recent trends in participation and outcomes. Youth and Society, 26, 194-222.

Lang, F. H., Forbes, J. F., Murray, G. D., et al (1997) Service provision for people with schizophrenia. I: Clinical and economic perspective. British Journal of Psychiatry, I7I, 159-164.

Laugharne, R., Byford, S., Barber, J. A., et al (2002) The effect of alcohol consumption on cost of care in severe psychotic illness: a report from the UK700 study. Acta Psychiatrica Scandinavica, 106, 24I-246.

Lehman, A. F. \& Steinwachs, D. M. (1998) Translating research into practice: the Schizophrenia Patient Outcomes Research Team (PORT) treatment recommendations. Schizophrenia Bulletin, 24, I-10.

Lumley, T., Diehr, P., Emerson, S., et al (2002) The importance of the normality assumption in large public health data sets. Annual Review of Public Health, 23, 15I-169.

McCrone, P., Johnson, S. \& Thornicroft, G. (2002) Predicting the costs of community care for individuals with severe mental illness in South London. Schizophrenia Bulletin, 27, 653-660.

McGorry, P. D. \& Edwards, J. (1998) The feasibility and effectiveness of early intervention in psychotic disorders: the Australian experience. International Clinical Psychopharmacology, 13 (suppl I) S47-S52.

McGorry, P. D., Yung, A. R., Phillips, L. J., et al (2002) Randomized controlled trial of interventions designed to reduce the risk of progression to first-episode psychosis in a clinical sample with subthreshold symptoms. Archives of General Psychiatry, 59, 921-928.

McGuffin, P., Farmer, A. \& Harvey, I. (1991) A polydiag nostic application of operational criteria in studies of psychotic illness. Development and reliability of the OPCRIT system. Archives of General Psychiatry, 48, 764-770.

\section{CLINICAL IMPLICATIONS}

- The identification of medium-term cost predictors such as non-completion of high-school education, age at illness onset and chronicity of illness course highlights the pervasive vulnerability within this group. Appropriate early intervention programmes for psychosis need to be developed and their cost-effectiveness evaluated from both government and societal perspectives.

- Assertive evidence-based rehabilitation and supported employment programmes need to be implemented and their cost-effectiveness evaluated in reducing the considerable disability that exists in social and occupational functioning.

- Further research is needed to determine whether pre-psychotic interventions can delay illness onset and the extent to which a chronic deteriorating illness trajectory is amenable to intervention.

\section{LIMITATIONS}

- The sample was predominantly urban and comprised people with psychotic disorders in relatively recent contact with health services. Other predictors may have emerged in a broader sample.

- Cost estimates were based on self-reports of service and resource utilisation, conservative unit prices and, in some instances, assumed standard quantities.

- Cross-sectional data were used, with a limited assessment of indirect costs. Prospective monitoring of actual costs could reveal different patterns of association with premorbid, psychosocial and clinical variables.

VAUGHAN J. CARR, FRANZCP, TERRY J. LEWIN, BCom(Psych) Hons, Centre for Mental Health Studies, University of Newcastle and Hunter Area Health Service; AMANDA L. NEIL, Grad Dip (Ec), Centre for Clinical Epidemiology and Biostatistics, University of Newcastle; SEAN A. HALPIN, BA(Psych) Hons, Centre for Mental Health Studies, University of Newcastle and Hunter Area Health Service; SCOTT HOLMES, PhD, Graduate School, University of Newcastle, Australia

Correspondence: Professor Vaughan J. Carr, Centre for Mental Health Studies, University of Newcastle, Callaghan, NSW 2308, Australia. Tel: 6I 2 492466I0; fax: 6I 2 49246608; e-mail:

Vaughan.Carr@hunter.health.nsw.gov.au

(First received 14 August 2003, final revision 5 January 2004, accepted 19 January 2004)

Mensch, B. S. \& Kandel, D. B. (1988) Dropping out of high school and drug involvement. Sociology of Education, 6I, 95-113.

Moscarelli, M., Capri, S. \& Neri, L. (1991) Cost evaluation of chronic schizophrenic patients during the first 3 years after the first contact. Schizophrenia Bulletin, 17, 421-426.

Rund, B. J. \& Ruud, T. (1999) Costs of services for schizophrenic patients in Norway. Acta Psychiatrica Scandinavica, 99, 120-125.

Suleiman,T. G., Ohaeri, J.U., Lawal, R. A., et al (1997) Financial costs of treating out-patients with schizophrenia in Nigeria. British Journal of Psychiatry, 171, 364-368.

Twamley, E. W., Jeste, D. V. \& Lehman, A. F. (2003) Vocational rehabilitation on schizophrenia and other psychotic disorders. A literature review and metaanalysis of randomized controlled trials. Journal of Nervous and Mental Disease, I9I, 5I5-523.

Van Oel, C. J., Sitskoorn, M. M., Cremer, M. P. M., et al (2002) School performance as a premorbid marker for schizophrenia: a twin study. Schizophrenia Bulletin, 28, $401-4 \mid 4$

Voruganti, L., Heslegrave, R., Awad, A., et al (1998) Quality of life measurement in schizophrenia: reconciling the quest for subjectivity with the question of reliability. Psychological Medicine, 28, 165-172.

Warner, R. (2003) How much of the burden of schizophrenia is alleviated by treatment? British Journal of Psychiatry, 183, 375-376.

Weinstein, M. C., Siegel, J. E., Garber, A. M., et al (1997) Productivity costs, time costs and health-related quality of life: a response to the Erasmus Group. Health Economics, 6, 505-510.

Wing, J. K., Babor, T., Brugha, T., et al (1990) SCAN: schedules for clinical assessment in neuropsychiatry. Archives of General Psychiatry, 47, 589-593.

World Health Organization (1988) WHO Psychiatric Disability Assessment Schedule (WHO/DAS). Geneva:WHO

World Health Organization (1992) Tenth Revision of the International Classification of Diseases and Related Health Problems (ICD-10). Geneva: WHO 